\title{
Sodium Pump Na+/K+ ATPase Subunit a1 Targeted Positron Emission Tomography Imaging of Hepatocellular Carcinoma in Mouse Models
}

\section{Si Tang}

Sun Yat-Sen University Cancer Prevention and Treatment Center: Sun Yat-sen University Cancer Center XiaoChun Yang

Sun Yat-Sen University Cancer Prevention and Treatment Center: Sun Yat-sen University Cancer Center Chao Zhou

Sun Yat-Sen University Cancer Prevention and Treatment Center: Sun Yat-sen University Cancer Center Yan Mei

Sun Yat-Sen University Cancer Prevention and Treatment Center: Sun Yat-sen University Cancer Center JiaCong Ye

Sun Yat-Sen University Cancer Prevention and Treatment Center: Sun Yat-sen University Cancer Center

XiaoFei Zhang

Sun Yat-Sen University Cancer Prevention and Treatment Center: Sun Yat-sen University Cancer Center

\section{GuoKai Feng}

Sun Yat-Sen University Cancer Prevention and Treatment Center: Sun Yat-sen University Cancer Center

\section{WeiGuang Zhang}

Sun Yat-Sen University Cancer Prevention and Treatment Center: Sun Yat-sen University Cancer Center

\section{Xu Zhang}

Sun Yat-Sen University Cancer Prevention and Treatment Center: Sun Yat-sen University Cancer Center Wei Fan ( $\square$ fanwei@sysucc.org.cn )

Sun Yat-Sen University Cancer Prevention and Treatment Center: Sun Yat-sen University Cancer Center https://orcid.org/0000-0001-5418-1714

\section{Research Article}

Keywords: NKAa1, PET, Hepatocellular carcinoma, Peptide

Posted Date: February 16th, 2021

DOI: https://doi.org/10.21203/rs.3.rs-204772/v1

License: (c) (1) This work is licensed under a Creative Commons Attribution 4.0 International License. Read Full License 


\section{Abstract}

Purpose: Positron emission tomography (PET) imaging was not efficiently used in early diagnosis of hepatocellular carcinoma (HCC) due to lack of appropriate tracers. Sodium pump $\mathrm{Na}+\mathrm{K}+\mathrm{ATPase}$ subunit a1 (NKAa1) emerges to be a prognostic and diagnostic biomarker of HCC. Here we investigated the feasibility of ${ }^{18}$ F-ALF-NOTA-S3, a PET tracer based on an NKAa1 peptide, to detect small HCC.

Methods: GEPIA database was searched to obtain the expression characteristics of NKAa1 in HCC and its relationship with the prognosis. PET/CT was performed in orthotopic, diethylnitrosamine (DEN)-induced and genetically engineered HCC mouse models to evaluate the use of ${ }^{18} \mathrm{~F}$-ALF-NOTA-S3 to detect HCC lesions.

Results: NKAa1 is overexpressed in early HCC with a high positive rate and correlates with poor survival. In orthotopic, DEN-induced and genetically engineered HCC mouse models, PET/CT imaging showed high accumulation of ${ }^{18} \mathrm{~F}$-ALF-NOTA-S3 in the tumor. The tumor-to-liver ratios are $2.56 \pm 1.02,4.41 \pm 1.09$ and $4.59 \pm 0.65$ respectively. Upregulated NKAa1 expression in tumors were verified by immunohistochemistry. Furthermore, ${ }^{18} \mathrm{~F}$-ALF-NOTA-S3 has the ability to detect small HCC lesions with diameters of $2-5 \mathrm{~mm}$.

Conclusion: NKAa1 may serve as a suitable diagnostic and prognostic biomarker for HCC. ${ }^{18}$ F-ALF-NOTAS3 shows great potential for PET imaging of HCC.

\section{Introduction}

Liver cancer is a major health threat and results in huge disease burden worldwide. It ranks sixth for cancer incidence, fourth for cancer deaths and is second leading cause of cancer years of life lost globally in 2015 (Fitzmaurice et al. 2017). Hepatocellular carcinoma (HCC) accounts for 75 85\% cases of liver cancer (Bray et al. 2018). Treatment for HCC patients diagnosed at an early stage (BCLC stage 0 to A) can lead to an average survival of 60 months. However, Patients with HCC who have advanced or end-stage disease (BCLC stage $C$ and $D$ ) at diagnosis have a poor prognosis, with expected median survival time of 6-8 months (stage C) and 3-4 months (stage D) (EASL Clinical Practice Guidelines: Management of hepatocellular carcinoma 2018). Therefore, early diagnosis is one of the keys to improving survival for HCC patients.

Current imaging techniques for clinical diagnosis of liver cancer include ultrasound, computed tomography (CT) and magnetic resonance imaging (MRI). The detection performance of these anatomically based imaging techniques closely correlates with tumor size. $\mathrm{HCC} \geq 2 \mathrm{~cm}$ in diameter can be accurately localized (approximately $100 \%$ ) by MRI and CT-enhanced scanning. However, for small HCC $1-2 \mathrm{~cm}$ in diameter, the sensitivity of MRI and CT-enhanced scanning gradually decreases, with MRI decreasing by $45-80 \%$ and CT decreasing by $40-75 \%$ (Ronot et al. 2016). Moreover, MRI and CT-enhanced imaging cannot detect extrahepatic lesions (Bellissimo et al. 2015), and lack the ability to predict 
response to treatment. Positron emission tomography (PET) enables whole-body imaging based on function and metabolism. $\beta-2-\left[{ }^{18} \mathrm{~F}\right]$-Fluoro-2-deoxy-D-glucose $\left({ }^{18} \mathrm{~F}-\mathrm{FDG}\right)$ is the most widely used tracer for PET imaging. Yet in more than half cases of HCC, significant uptake of FDG was not observed (CastillaLièvre et al. 2016). Most well differentiated HCCs are negative for ${ }^{18}$ F-FDG PET. Thus, FDG PET-scan is not recommended for early diagnosis of HCC. The detection rates of some other existing tracers (such as 11C-choline) are also unsatisfactory (Chotipanich et al. 2016). Nowadays, few imaging approaches are perfect for the early diagnosis of small HCC. As a result, more effective imaging methods are needed to improve the diagnosis of HCC.

$\mathrm{Na}+$ /K+-ATPase (NKA), an enzyme present in almost all higher eukaryotic cell membranes, is a member of the P-type ATPase family. Under physiological conditions, NKA plays a key role in cellular ion transport, metabolism, migration and signal transduction (Cui and Xie 2017; Pressley 1996). NKA contains 2 subunits, the $a$ (including a1 a4) catalytic subunit and the $\beta$ (including $\beta 1 \sim \beta 3$ ) regulatory subunit (Blanco and Mercer 1998; Levenson 1994). Among them, the a1 subunit, NKAa1, is abnormally overexpressed in a variety of cancers (Mijatovic et al. 2007a; Seligson et al. 2008; Winnicka et al. 2008; Xu et al. 2010), and considered a potential target for anticancer drug development (Alevizopoulos et al. 2014; Mijatovic et al. 2007b). NKAa1 was also found to be overexpressed in HCC, and knockdown of this gene led to cell-cycle arrest and apoptosis in HCC cells (Zhuang et al. 2015). These studies suggest that targeting NKAa1 may be of help to the treatment of HCC. Consequently, the development of NKAa1targeted imaging methods may be used for both detection of HCC and assessment of related therapies in the future. Our previous studies used phage display to screen a breast cancer-target peptide of NKAa1, namely S3. The specific binding of S3 peptide to NKAa1 was validated. PET imaging was successfully performed in breast cancer mouse models after this peptide radio-labeled and the tracer's in vitro and in vivo stability verified (Wang et al. 2018). We believe this targeted S3 peptide could serve as a new approach for the imaging of HCC.

In this study, we first investigated the overexpression of NKAa1 in $\mathrm{HCC}$ based on public databases and established literature. Then, we performed PET/CT imaging using the tracer ${ }^{18} \mathrm{~F}-\mathrm{ALF}$-NOTA-S3 (dubbed ${ }^{18} \mathrm{~F}$-S3 for short) in orthotopic implantation HCC mice and validated the feasibility of this targeted peptide in PET imaging of HCC. We subsequently performed PET/CT imaging on chemically induced HCC mice, as this model has much in common with human liver cancer in terms of morphology and biochemical features (He et al. 2015). Lastly, we use genetically engineered HCC mice to further confirmed the feasibility of the tracer detecting small HCC lesions.

\section{Materials And Methods}

\subsection{Cells and mice}

The HCC cell line used in this study is HCC-LM3 cells purchased from the American Type Culture Collection (ATCC). This cell line was cultured with DMEM containing $10 \%$ foetal bovine serum. BALB/C mice and C57BL6 mice in this study were purchased from Vital River, Charles River Laboratories China 
(Beijing, China). The animal experiments were accredited through the Institutional Animal Care and Use Committee (IACUC) in Sun Yat-sen University Cancer Center.

To facilitate monitoring of tumorigenesis, HCC-LM3 cells were stably transfected with luciferase to form HCC-LM3-luciferase cells. Anesthetized mice were surgically exposing their livers in a sterile environment. Then the HCC-LM3-luciferase cells were injected orthotopically to the left lobe of the liver under direct observation. One week after surgery, the tumorigenesis of mice was confirmed by bioluminescence imaging.

For the construction of the chemical induced HCC mouse model, a single dose of diethynitrosamine (DEN) $(200 \mathrm{mg} / \mathrm{kg})$ was administered into male C57BL6 mice to induce HCC. Mice were scanned via MRI to verify the presence of HCC lesions in the livers 36 weeks after injection.

$\mathrm{H} 11^{\mathrm{LNL}-M y c}$ knock-in HCC mouse model was developed by Shanghai Model Organisms Center, Inc. This model was generated by CRISPR/Cas9 system in C57BL/ $6 \mathrm{~J}$ mouse background. Technical details are the same as described in previous literature (Feng et al. 2019). F0 mice were crossed with C57BL/6 J mice to gain $\mathrm{H} 11^{\mathrm{LNL}-M y c}$ heterozygous mice. $\mathrm{H} 11^{\text {LNL-Myc; }}$; Alb-Cre double positive mice used in this study were generated by crossing $\mathrm{H} 11^{\mathrm{LNL}-M y c}$ heterozygous mice with Alb-Cre transgenic mice.

\subsection{Preparation of ${ }^{18} \mathrm{~F}-\mathrm{S} 3$}

NOTA-CSISSLTHC (NOTA-S3) were synthesized by the Chinese Peptide Company (Hangzhou, China) as following procedure: NOTA bis(tBu)ester and full covered peptide was once dissolved in $\mathrm{N}, \mathrm{N}-$ Dimethylformamide (DMF). Then 2-(7-Azabenzotriazol-1-yl)-N,N,N',N'-tetramethyluronium hexafluorophosphate (HATU) and N-methylmorpholine (NMM) were introduced to the reaction mixture. The response mixture was stirred at room temperature for 3 hours, then workup to supply the NOTAmodified peptide which was cleavage with the aid of tallow fatty acid to provide the crude peptide. Last, it was purified via HPLC to provide final product. The peptide is available as a freeze-dried powder freely soluble in saline solution. Then, the radiolabeling reaction procedure was as follows: the vials containing $8 \mathrm{nmol}$ of NOTA-S3 and $6 \mathrm{nmol}$ of $\mathrm{AlCl}_{3} \cdot 6 \mathrm{H}_{2} \mathrm{O}$ was added with $65 \mu \mathrm{l}$ of ${ }^{18} \mathrm{~F}$ - (about $10 \mathrm{mCi}$ ) in DI water, 3 $\mu \mathrm{l}$ of acetic acid and $324 \mu \mathrm{l}$ acetonitrile, heated at $100^{\circ} \mathrm{C}$ for $10 \mathrm{~min}$. After the vial was cooled to room temperature, $10 \mathrm{ml}$ of water was added to dilute the mixture. Then the mixture was trapped on a Varian Bond Elut C18 column (100 mg) using a 20-ml syringe. After washed with $10 \mathrm{ml}$ of water, the $\mathrm{C} 18 \mathrm{column}$ was eluted with $0.4 \mathrm{ml}$ of ethanol twice. The solution was dried by a vacuum dryer. The final product ${ }^{18} \mathrm{~F}-$ ALF-NOTA-S3 was dissolved with saline solution before injection.

\subsection{PET/CT imaging}

The mice were fasted from food but not water for 6 hours prior to the imaging experiments. Mice were anesthetized with $1.2 \%$ tribromoethanol solution at a dose of $250 \mathrm{mg} / \mathrm{kg}$ and then intravenously injected with approximately $60 \sim 100 \mu \mathrm{Ci}(2.22 \sim 3.7 \mathrm{MBq})$ of ${ }^{18} \mathrm{~F}$-S3. PET/CT scans were performed after 1 1.5 hours of circulation. While waiting, the mice were kept warm by an electric blanket. After acquiring the 
images, regions of interest (ROIs) were drawn on the tumor and normal organs. The maximum uptake value was calculated automatically, and the tumor boundary was differentiated by a low threshold of $30 \%$ of the maximum uptake. The radiation intensity of tumor and normal organs was calculated as percentage of the injected dose per gram $(\% \mathrm{ID} / \mathrm{g})$.

\subsection{Biodistribution}

In biodistribution analysis from static PET/CT images, ROls were placed over the tumor and major organs (liver, heart, lung, intestine, kidneys, brain and muscle) through layered sketching with the assistance of corresponding CT images. The results were expressed in percentage of the injected dose per gram $(\% \mathrm{ID} / \mathrm{g})$.

\subsection{Immunohistochemistry}

Samples were fixed with formalin, embedded in paraffin, sectioned, and stained with H\&E in accordance to standard histopathological techniques. Paraffin sections were de-paraffinized with xylene and rehydrated. The sections were processed in citrate buffer $(\mathrm{pH} \mathrm{6})$ and microwaved for antigen retrieval. To block nonspecific binding, the sections were incubated with $1 \%$ bovine serum albumin after a treatment with $3 \%$ hydrogen peroxide in methanol and quenching the endogenous peroxidase activity. The sections were then stained with an NKAa1 antibody (Abcam, M7-PB-E9, 1:150) for $12 \mathrm{~h}$ at $4{ }^{\circ} \mathrm{C}$. After washing, the sections were incubated with an HRP-conjugated polyclonal secondary antibody (1:200). The sections were immersed in 3-amino-9-ethyl carbazole, counterstained with 10\% Mayer's hematoxylin, dehydrated, and mounted in crystal mount.

\subsection{Statistical analysis}

Statistical analyses were performed using R 4.0.3. Data were presented as means $\pm S D$. Means were

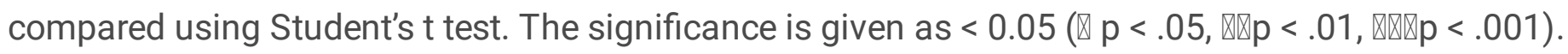

\section{Results}

\subsection{Overexpression and prognostic value of NKAa1 in HCC}

To investigate the overexpression of NKAa1 and its positive rate in HCC, we first analyzed the GEPIA database (http://gepia.cancer-pku.cn/), a website for cancer and normal gene expression profiling and interactive analyses. Retrieval results showed that NKAa1 expression is significantly elevated in LIHC (Liver hepatocellular carcinoma) compared to normal liver tissue (Fig. 1a). In a recent research by Jiang et al., paired tumors and adjacent normal liver tissues of HBV related early-stage HCC were characterized (Jiang et al. 2019). According to the original gene expression profiles of HCC tumor and non-tumor quantified, in which NKAa1 expression was included in 35 pairs of tumor/none-tumor samples, we observed approximately $94 \%$ (33 out of 35) of early-stage HCC with NKAa1 overexpression compared with paired normal liver tissues at the RNA level (Fig. 1b). Finally, we linked NKAa1 to the prognosis of HCC by analyzing the GEPIA database. In accordance with a previous study by Zhuang et al.(Zhuang et 
al. 2015), we observed that high NKAa1 correlates with poor overall survival (Fig. 1c) and disease-free survival (Fig. 1d). To sum up, we confirmed the extreme high positive rate of NKAa1 overexpression in clinical early-stage HCC. NKAa1 may serve as a potential diagnostic biomarker for targeted molecular imaging of early-stage HCC.

3.2. PET imaging with ${ }^{18} \mathrm{~F}-\mathrm{S} 3$ in genetically engineered, DEN induced and orthotopic HCC mouse models and biodistribution of ${ }^{18} \mathrm{~F}-\mathrm{S} 3$ in vivo.

We radiolabeled the NKAa1-targeted S3 peptide with a radionuclide fluorine-18 $\left({ }^{18} \mathrm{~F}\right)$ to form a PET tracer ${ }^{18} \mathrm{~F}-\mathrm{S} 3$ (Fig. 2) according to our previous report(Wang et al. 2018), in which the stability and time activity curves of this tracer were also characterized. The radiochemical yield of ${ }^{18} \mathrm{~F}$-S3 was about $35 \%$ without decay correction. We then performed PET/CT imaging with ${ }^{18} \mathrm{~F}-\mathrm{S} 3$ in orthotopic, DEN-induced and genetically engineered $\mathrm{HCC}$ mice respectively.

Seven days after the orthotopic implantation surgery, fluorescence imaging (Fig. 3a) was performed for monitoring HCC tumorigenesis. The diameters of the orthotopically implanted HCC-LM3 tumors were in the range of $2 \mathrm{~mm}$ to $5 \mathrm{~mm}$, which were then confirmed by autopsy (Fig. $3 \mathrm{~b}$ ) after the PET imaging experiment. In an orthotopic HCC-LM3-luciferase liver tumor bearing mouse, representative PET/CT imaging and maximum intensity projection (MIP) image (Fig. 3c) showed high and specific tumor uptake of ${ }^{18} \mathrm{~F}$-S3. In whole-body PET imaging, tumor uptake was second only to the urinary system, and the lesion can be finely distinguished. Quantification indicated that tumor uptake of ${ }^{18} \mathrm{~F}-\mathrm{S} 3$ was $3.02 \pm 0.59$ $\% \mathrm{ID} / \mathrm{g}$ and background liver uptake was $1.28 \pm 0.34 \% \mathrm{ID} / \mathrm{g}$ (Fig. $3 \mathrm{~d}$ ), leading to a tumor-to-liver ratio of $2.56 \pm 1.02$.

In a DEN-induced HCC mouse (Fig. 4a), representative PET/CT imaging with maximum intensity projection (Fig. 4b) showed ${ }^{18} \mathrm{~F}$-S3 was highly accumulated in the tumor. We quantitatively calculated the tumor uptake of ${ }^{18} \mathrm{~F}$-S3 was $4.61 \pm 0.44 \% \mathrm{ID} / \mathrm{g}$ and normal liver uptake was $1.08 \pm 0.24 \% \mathrm{ID} / \mathrm{g}$ (Fig. $4 \mathrm{c}$ ), resulting in a tumor-to-liver ratio of $4.41 \pm 1.09$. We mapped the organs by layers and quantified their uptake of ${ }^{18} \mathrm{~F}$-S3(Fig. $4 \mathrm{~d}$ ). Tumor uptake of ${ }^{18} \mathrm{~F}$-S3 was not only higher than the liver background, but also significantly higher than other major organs except for the kidney. Immunohistochemistry showed significant overexpression of NKAa1 in tumor tissues compared to adjacent normal liver in DEN-induced HCC (Fig. 4e).

Before we used $\mathrm{H} 11^{\mathrm{LNL}-M y c}$ genetically engineered mice for imaging, the tumorigenesis of these mice had been monitored by primovist (Gd-EOB-DTPA) -enhanced MRI (Fig. 5a), which had shown an approximate $3 \mathrm{~mm} \times 6 \mathrm{~mm}$ tumor near the right-lower margin of the liver in a representative mouse. PET/CT and MIP imaging showed that ${ }^{18} \mathrm{~F}$-S3 was highly accumulated in the tumor (Fig. $5 \mathrm{~b}$ ). In quantitative calculation, the tumor and liver uptake of ${ }^{18} \mathrm{~F}$-S3 were $4.45 \pm 0.97 \% \mathrm{ID} / \mathrm{g}$ and $0.86 \pm 0.09 \% \mathrm{ID} / \mathrm{g}$ respectively (Fig. $5 \mathrm{c}$ ). The tumor-to-liver ratio is $4.59 \pm 0.65$. We dissected the liver of this mouse intact and obtained tumor tissue (Fig. 5d). Immunohistochemistry revealed that in genetically engineered mice of liver cancer, 
NKAa1 was significantly overexpressed in tumor tissue compared to adjacent normal liver tissue (Fig. $5 e)$.

\section{Discussion}

Difficulty in early diagnosis of HCC is one of the major obstacles to improving the prognosis of liver cancer. PET imaging modalities are widely used in the field of oncology for improving the diagnosis and management of cancer patients, because of their unlimited penetration depth, high sensitivity, and quantitative imaging capabilities. However, efficient use of PET in the early diagnosis of liver cancer has been hampered by the lack of appropriate tracers. Prior to this study, several antibody-based PET imaging agents specifically targeting $\mathrm{HCC}$ had been proposed, such as ${ }^{89} \mathrm{Zr}$-conjugated monoclonal antibody against GPC3 (Sham et al. 2014) and CD146 (Hernandez et al. 2016). However, peptide-based tracers may have an advantage over them in PET imaging because of their small size, low immunogenicity, sufficient capillary permeability and rapid clearance from non-target tissues. Evidence from established literature in the past decade suggests that NKAa1 may be an important target in the treatment of HCC (Jin et al. 2020; Xu et al. 2010; Zhuang et al. 2015). In this study, we furtherly demonstrated the overexpression of NKAa1 in HCC. Furthermore, high NKAa1 expression is significantly associated with worse prognosis in HCC. We then applied the PET imaging agent, ${ }^{18} \mathrm{~F}-\mathrm{S} 3$ to provide high-quality imaging in small liver cancers. Since there have been evidence that the cardiac glycosides (CGs) digoxin, digitoxin and ouabain, which directly inhibit NKAa1 function, could exhibite anticancer effects (Kim et al. 2016), it is promising that this study may help integrate the diagnosis and treatment of HCC in the future.

Different animal models have their advantages and disadvantages in representing the biological characteristics of HCC and mimicking the human tumor microenvironment (He et al. 2015). For this reason, we used multiple animal models to validate the feasibility of PET imaging of HCC based on the NKAa1-targeted peptide S3. In orthotopic implantation HCC models, by monitoring the growth of the implant tumor, we have achieved imaging of very small liver cancers, which demonstrated the strong potential of ${ }^{18} \mathrm{~F}-\mathrm{S} 3$ in early imaging diagnosis of small liver cancer. The gene expression patterns in HCC in DEN-induced mouse liver cancers were most similar to those of the poorer survival group of human HCC (Lee et al. 2004). In this type of HCC mice, we also observed favorable imaging results. Possibly due to the larger tumor and clearer borders, the tumor-to-liver ratio is higher in DEN-induced mice compared to orthotopic implantation HCC mice. The good tumor specificity of this tracer and its low toxicity to other organs is supported by the biodistribution experiments performed in DEN mice. Genetically engineered HCC mouse models are excellent for studies concerning on the molecular mechanism of liver cancer development. In this model, we could obtain MR-equivalent detection efficiency for small HCC lesions. Considering that PET/CT can simultaneously scan the whole body for early metastases, this result could be meaningful. Of particular note, the detection of lesions with a longest diameter of less than 5-6 mm was achieved with ${ }^{18} \mathrm{~F}-\mathrm{S} 3$ in multiple mouse models. In multiple models, we verified by immunohistochemistry that NKAa1 expression is upregulated in tumors, albeit to different extents. The most significant differences of expression were observed in the genetically engineered HCC mouse. 
Nevertheless, this study has several limitations. First, quantitative analysis in this study shows that the affinity of ${ }^{18} \mathrm{~F}$-S3 is relatively low compared to the integrin-a6 targeted RWY peptide we previously reported in PET imaging of HCC (Feng et al. 2019). This peptide may also be less well prepared than other widely used peptides such as RGD. This is partly because the above-mentioned peptides have been intensively studied in the PET and MR imaging of a variety of cancers (Beer et al. 2008; Zhang et al. 2020; Zhu et al. 2012), and their structures and affinities have been optimized several times. Second, the biodistribution analysis in this study was based on the ROIs mapped in PET images, which could be less accurate than direct measurements of the radioactive counts of extracted organs. Last, in this experiment, little morphologic changes can be identified by the simultaneous CT images and we had to rely on MR, bioluminescence imaging, and autopsy to confirm the tumor before or after PET imaging. This may lead to mild inaccuracies in locating the lesion and the assessment of tumor size in PET images. Although these limitations do not undermine the basic conclusions of this study, they will be improved in our future research.

To sum up, we constructed various types of mouse models and used them to validate the feasibility of ${ }^{18} \mathrm{~F}-\mathrm{S} 3$ in PET imaging of $\mathrm{HCC}$ for the first time. Although the imaging results are promising, some limitations exist in this research. In order to promote the further application of this tracer in PET imaging, it needs to be modified and optimized for better affinity and specificity in future research. The safety and the proper radiation dose for imaging of ${ }^{18} \mathrm{~F}-\mathrm{S} 3$ in $\mathrm{HCC}$ should also be further explored.

\section{Conclusions}

In conclusion, NKAa1 may serve as a suitable diagnostic and prognostic biomarker for HCC. We reported the use of an NKAa1-targeted peptide-based PET tracer ${ }^{18} \mathrm{~F}$-S3 for the detection of HCC in three kinds of $\mathrm{HCC}$ mouse models. The high sensitivity of ${ }^{18} \mathrm{~F}$-S3 and its well-defined feasibility of detecting small HCCs have been demonstrated. ${ }^{18} \mathrm{~F}$-S3 shows good potential for the imaging of HCC, and it may serve as a new approach for diagnosis of early and small HCC.

\section{Abbreviations}

BCLC, Barcelona Clinic Liver Cancer

DEN, diethylnitrosamine

HCC, hepatocellular carcinoma

MIP, maximum intensity projection

$\mathrm{MRI}$, magnetic resonance imaging

NKAa1, Na+/K+ ATPase subunit a1 
PET/CT, positron emission tomography/computed tomography

$\mathrm{ROI}$, region of interest

\section{Declarations}

\section{Funding}

This work was supported by the National Natural Science Foundation of China (NSFC) (projects 81972531 and 82001855) \the China Postdoctoral Science Foundation (2019M663297), and the Fundamental Research Funds for the Central Universities 19ykpy174.

\section{Conflicts of interest/Competing interests}

The authors declare no conflict of interest.

\section{Ethics approval}

This study was approved by the Institutional Animal Care and Use Committee (IACUC) in Sun Yat-sen University Cancer Center.

\section{Consent to participate}

Not applicable.

\section{Consent for publication}

All authors approved of the manuscript and consented to its publication.

\section{Availability of data and material}

All data are included in the manuscript.

\section{Code availability}

Not applicable.

\section{Authors' contributions}

All authors contributed to the study conception and design. Material preparation, data collection and analysis were performed by ST, XCY and YM. ST, JCY, XFZ and GKF performed the experiments. The whole experiment was completed under the guidance of WGZ, XZ and WF. The first draft of the manuscript was written by ST and XCY. All authors commented on previous versions of the manuscript and approved the final manuscript. 


\section{References}

1. Alevizopoulos K, Calogeropoulou T, Lang F, Stournaras C (2014) Na+/K+ ATPase inhibitors in cancer Current drug targets 15:988-1000

2. Beer AJ et al. (2008) Comparison of integrin alphaVbeta3 expression and glucose metabolism in primary and metastatic lesions in cancer patients: a PET study using 18F-galacto-RGD and 18F-FDG Journal of nuclear medicine : official publication, Society of Nuclear Medicine 49:22-29 doi:10.2967/jnumed.107.045864

3. Bellissimo F, Pinzone MR, Cacopardo B, Nunnari G (2015) Diagnostic and therapeutic management of hepatocellular carcinoma World journal of gastroenterology 21:12003-12021 doi:10.3748/wjg.v21.i42.12003

4. Blanco G, Mercer RW (1998) Isozymes of the Na-K-ATPase: heterogeneity in structure, diversity in function The American journal of physiology 275:F633-650 doi:10.1152/ajprenal.1998.275.5.F633

5. Bray F, Ferlay J, Soerjomataram I, Siegel RL, Torre LA, Jemal A (2018) Global cancer statistics 2018: GLOBOCAN estimates of incidence and mortality worldwide for 36 cancers in 185 countries CA Cancer J Clin 68:394-424 doi:10.3322/caac.21492

6. Castilla-Lièvre MA et al. (2016) Diagnostic value of combining ${ }^{11} \mathrm{C}$-choline and ${ }^{1} \otimes \mathrm{F}-\mathrm{FDG}$ PET/CT in hepatocellular carcinoma European journal of nuclear medicine and molecular imaging 43:852-859 doi:10.1007/s00259-015-3241-0

7. Chotipanich C, Kunawudhi A, Promteangtrong C, Tungsuppawattanakit P, Sricharunrat T, Wongsa $P$ (2016) Diagnosis of Hepatocellular Carcinoma Using C11 Choline PET/CT: Comparison with F18 FDG, ContrastEnhanced MRI and MDCT Asian Pacific journal of cancer prevention : APJCP 17:35693573

8. Cui X, Xie Z (2017) Protein Interaction and Na/K-ATPase-Mediated Signal Transduction Molecules (Basel, Switzerland) 22 doi:10.3390/molecules22060990

9. EASL Clinical Practice Guidelines: Management of hepatocellular carcinoma (2018) J Hepatol 69:182-236 doi:10.1016/j.jhep.2018.03.019

10. Feng GK et al. (2019) Integrin alpha6 targeted positron emission tomography imaging of hepatocellular carcinoma in mouse models $\mathrm{J}$ Control Release 310:11-21 doi:10.1016/j.jconrel.2019.08.003

11. Fitzmaurice C et al. (2017) Global, Regional, and National Cancer Incidence, Mortality, Years of Life Lost, Years Lived With Disability, and Disability-Adjusted Life-years for 32 Cancer Groups, 1990 to 2015: A Systematic Analysis for the Global Burden of Disease Study JAMA Oncol 3:524-548 doi:10.1001/jamaoncol.2016.5688

12. He L, Tian DA, Li PY, He XX (2015) Mouse models of liver cancer: Progress and recommendations Oncotarget 6:23306-23322 doi:10.18632/oncotarget.4202

13. Hernandez R et al. (2016) CD146-targeted immunoPET and NIRF Imaging of Hepatocellular Carcinoma with a Dual-Labeled Monoclonal Antibody Theranostics 6:1918-1933 
doi:10.7150/thno.15568

14. Jiang $Y$ et al. (2019) Proteomics identifies new therapeutic targets of early-stage hepatocellular carcinoma Nature 567:257-261 doi:10.1038/s41586-019-0987-8

15. Jin X et al. (2020) Cinobufagin Triggers Defects in Spindle Formation and Cap-Dependent Translation in Liver Cancer Cells by Inhibiting the AURKA-mTOR-elF4E Axis The American journal of Chinese medicine 48:651-678 doi:10.1142/s0192415x20500330

16. Kim N et al. (2016) Cardiac glycosides display selective efficacy for STK11 mutant lung cancer Scientific reports 6:29721 doi:10.1038/srep29721

17. Lee JS, Chu IS, Mikaelyan A, Calvisi DF, Heo J, Reddy JK, Thorgeirsson SS (2004) Application of comparative functional genomics to identify best-fit mouse models to study human cancer Nature genetics 36:1306-1311 doi:10.1038/ng1481

18. Levenson R (1994) Isoforms of the Na,K-ATPase: family members in search of function Reviews of physiology, biochemistry and pharmacology 123:1-45 doi:10.1007/BFb0030902

19. Mijatovic T et al. (2007a) The alpha1 subunit of the sodium pump could represent a novel target to combat non-small cell lung cancers The Journal of pathology 212:170-179 doi:10.1002/path.2172

20. Mijatovic T, Van Quaquebeke E, Delest B, Debeir O, Darro F, Kiss R (2007b) Cardiotonic steroids on the road to anti-cancer therapy Biochim Biophys Acta 1776:32-57 doi:10.1016/j.bbcan.2007.06.002

21. Pressley TA (1996) Structure and function of the Na,K pump: ten years of molecular biology Mineral and electrolyte metabolism 22:264-271

22. Ronot M, Clift AK, Vilgrain V, Frilling A (2016) Functional imaging in liver tumours J Hepatol 65:10171030 doi:10.1016/j.jhep.2016.06.024

23. Seligson DB et al. (2008) Na,K-adenosine triphosphatase alpha1-subunit predicts survival of renal clear cell carcinoma The Journal of urology 179:338-345 doi:10.1016/j.juro.2007.08.094

24. Sham JG et al. (2014) Glypican-3-targeted 89Zr PET imaging of hepatocellular carcinoma Journal of nuclear medicine : official publication, Society of Nuclear Medicine 55:799-804 doi:10.2967/jnumed.113.132118

25. Wang $Q$ et al. (2018) Identification of a sodium pump $\mathrm{Na}(+) / \mathrm{K}(+)$ ATPase alpha1-targeted peptide for PET imaging of breast cancer J Control Release 281:178-188 doi:10.1016/j.jconrel.2018.05.019

26. Winnicka K, Bielawski K, Bielawska A, Surazyński A (2008) Antiproliferative activity of derivatives of ouabain, digoxin and proscillaridin A in human MCF-7 and MDA-MB-231 breast cancer cells Biological \& pharmaceutical bulletin 31:1131-1140 doi:10.1248/bpb.31.1131

27. Xu ZW, Wang FM, Gao MJ, Chen XY, Hu WL, Xu RC (2010) Targeting the Na(+)/K(+)-ATPase alpha1 subunit of hepatoma HepG2 cell line to induce apoptosis and cell cycle arresting Biological \& pharmaceutical bulletin 33:743-751 doi:10.1248/bpb.33.743

28. Zhang $Y$ et al. (2020) Integrin a6-Targeted Magnetic Resonance Imaging of Hepatocellular Carcinoma in Mice Mol Imaging Biol 22:864-872 doi:10.1007/s11307-019-01437-z 
29. Zhu Z et al. (2012) 99mTc-3PRGD2 for integrin receptor imaging of lung cancer: a multicenter study Journal of nuclear medicine : official publication, Society of Nuclear Medicine 53:716-722 doi:10.2967/jnumed.111.098988

30. Zhuang $L$ et al. (2015) Na+/K+-ATPase a1 subunit, a novel therapeutic target for hepatocellular carcinoma Oncotarget 6:28183-28193 doi:10.18632/oncotarget.4726

\section{Figures}


a

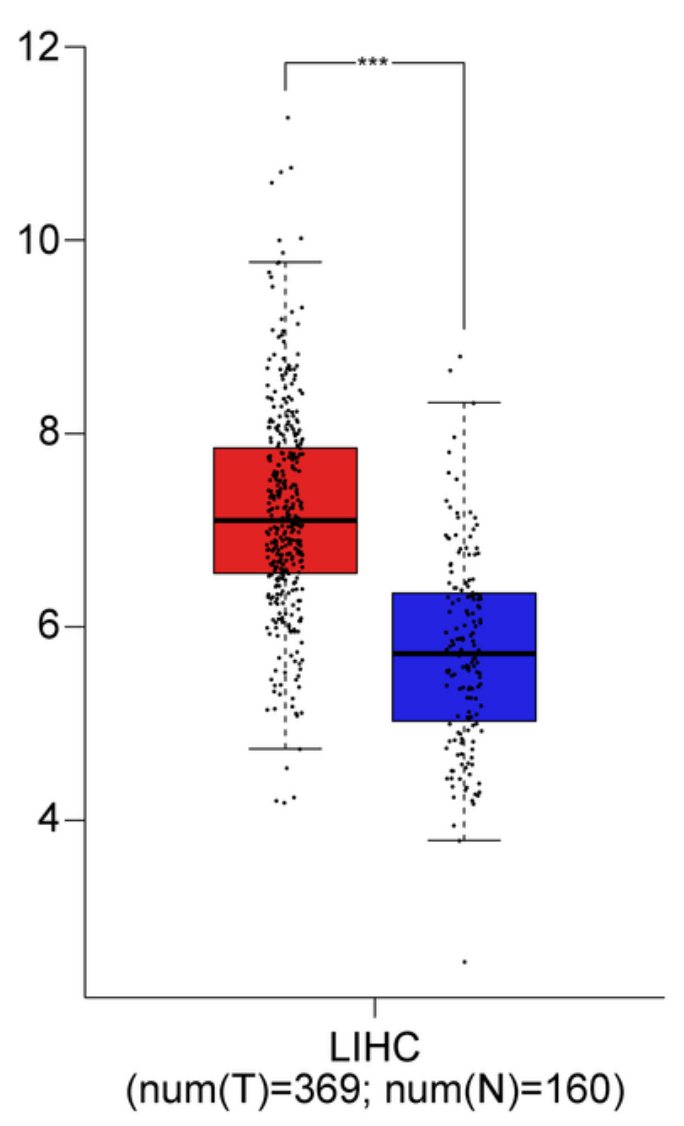

C

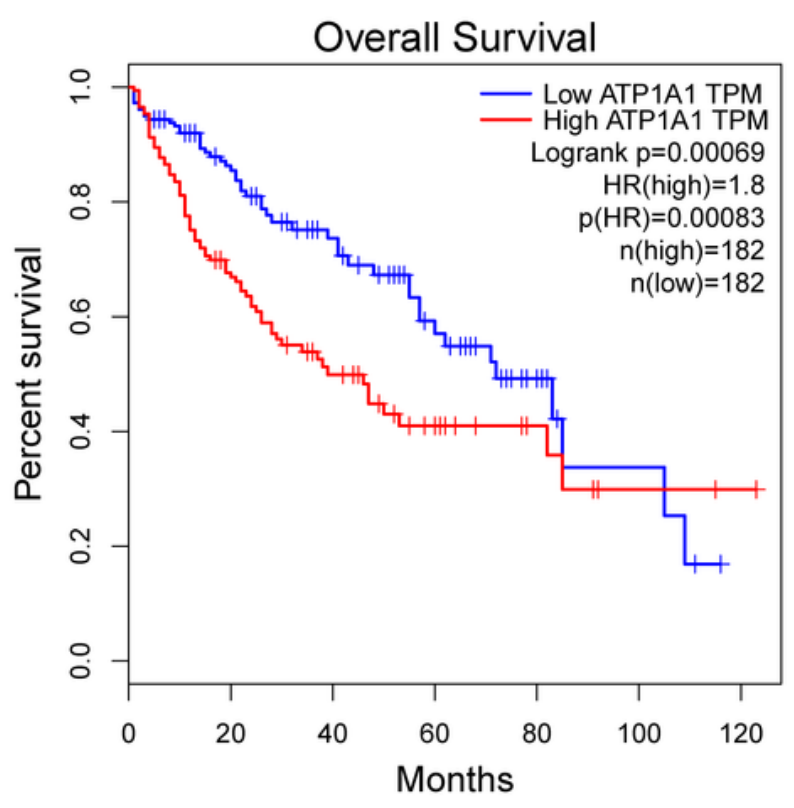

b

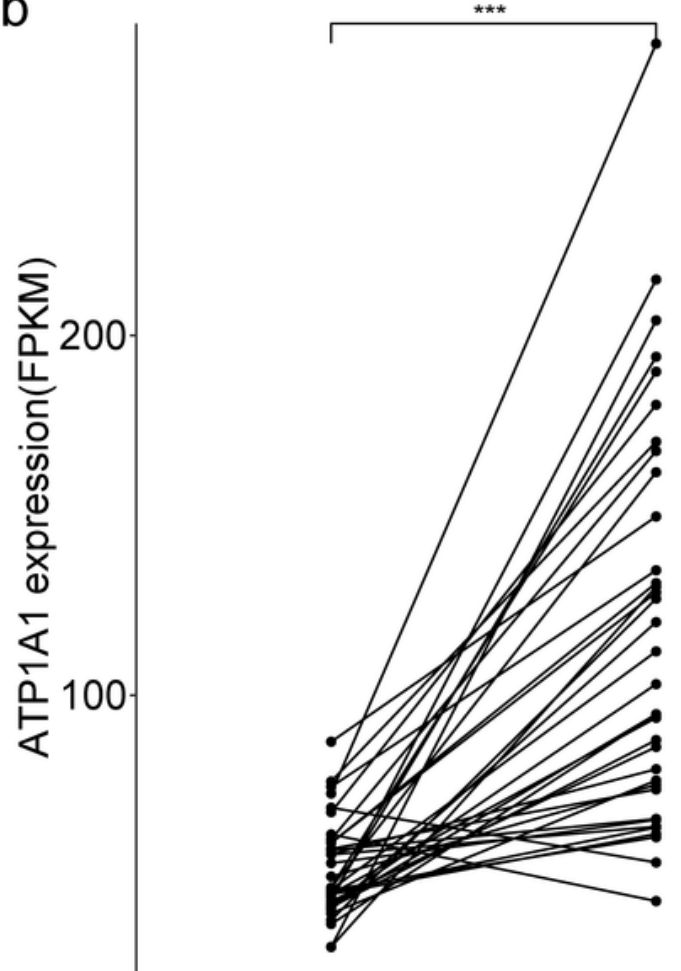

Non-tumor

Tumor

d

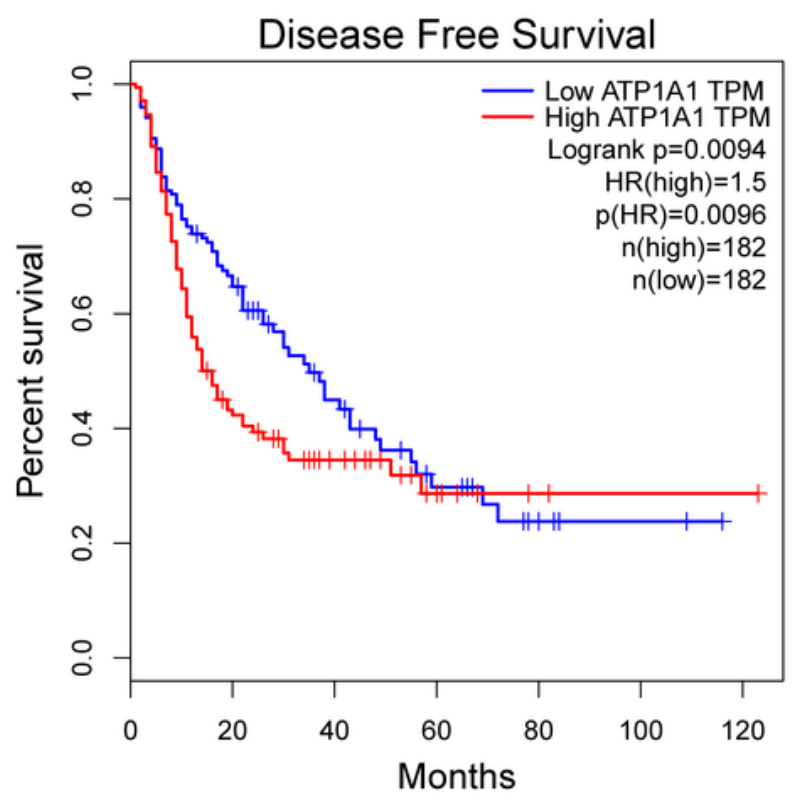

\section{Figure 1}

Overexpression and prognostic value of NKAa1 in HCC. (a) Boxplot of NKAa1 expression in LIHC tumor compared with normal tissue. Red: Tumor tissues. Blue: Normal liver tissues. Student's t test, ${ }^{\star \star \star} \mathrm{p}<$ 0.001. (b) $94 \%$ of HCC samples with ATP1A1 overexpression. Paired Student's t test, ${ }^{\star \star \star} \mathrm{p}<0.001$. High NKAa1 expression correlates with poor overall survival(c) and disease free (d) survival in HCC by 
analyzing the GEPIA database (http://gepia.cancer-pku.cn/). Gene: NKAa1; Datasets: LIHC; Group Cutoff: Median; Cutoff-High (\%): $50 \%$ and Cutoff-Low (\%): 50\%

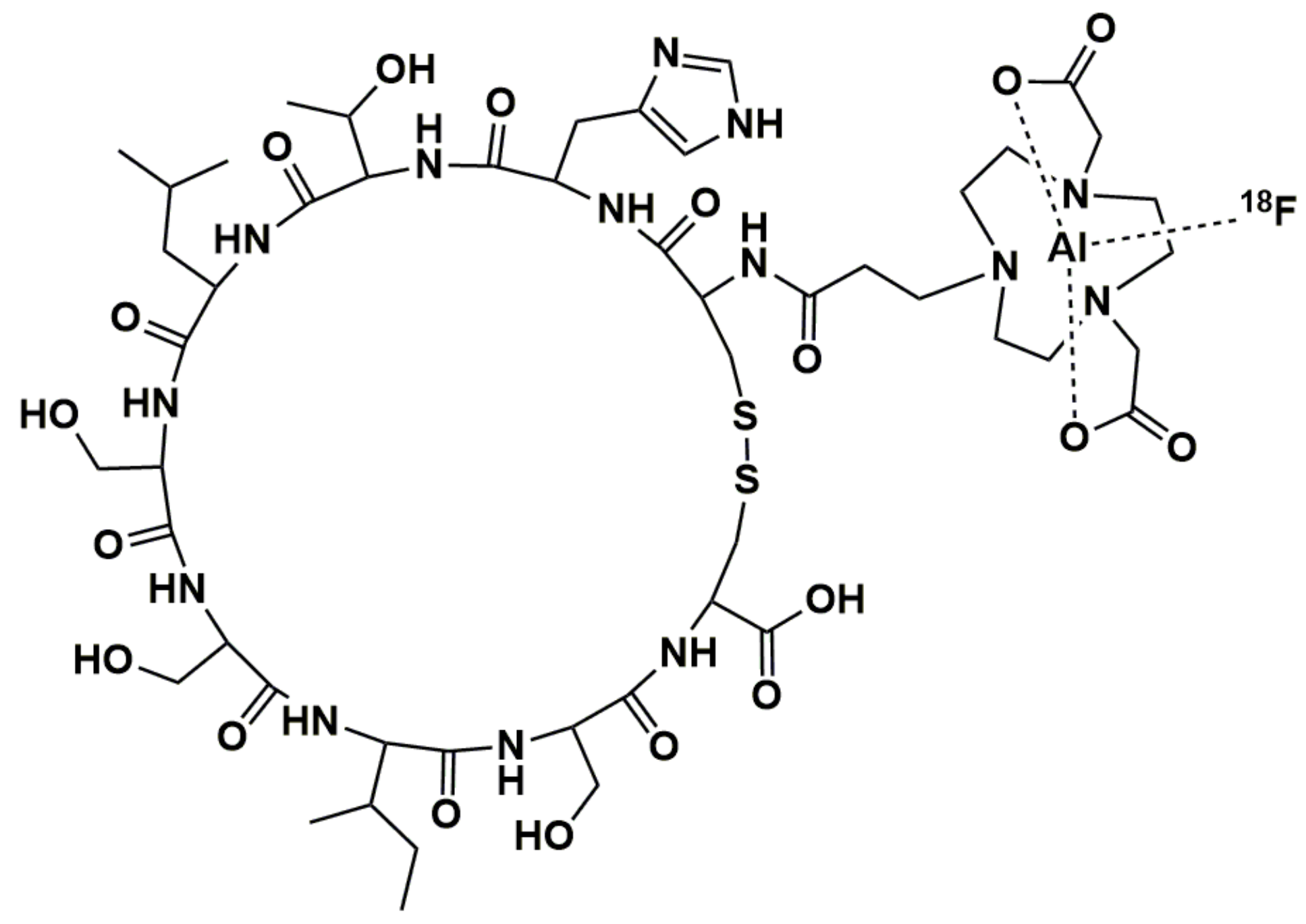

Figure 2

Chemical structure of 18F-ALF-NOTA-S3 
a
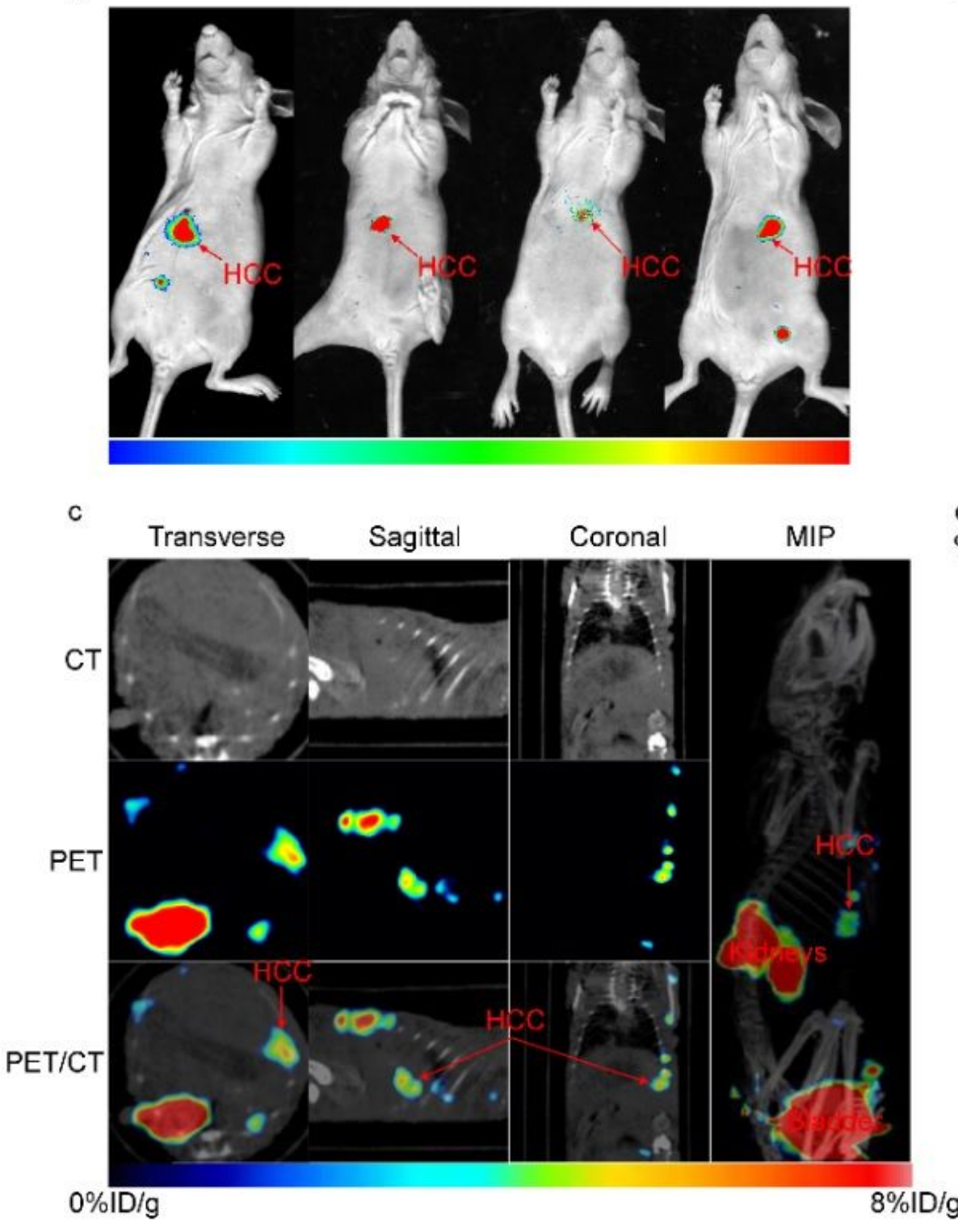

b

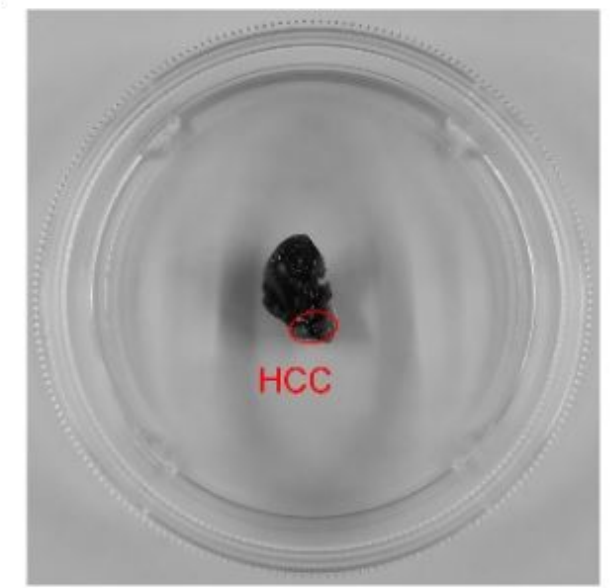

d



Figure 3

PET/CT imaging with 18F-S3 in HCC-LM3 orthotopic HCC animal models. (a) Bioluminescence imaging performed with luciferin in orthotopic HCC-LM3-luciferase mice. (b) Photograph of partial left lobe of a dissected liver. (c) Representative PET/CT imaging and MIP in an HCC-LM3 orthotopic HCC mouse. (d) Quantification of tumor and liver uptakes of 18F-S3. Student's t-test, $\otimes \mathbb{Q} p<.01, n=4$, mean \pm SD 

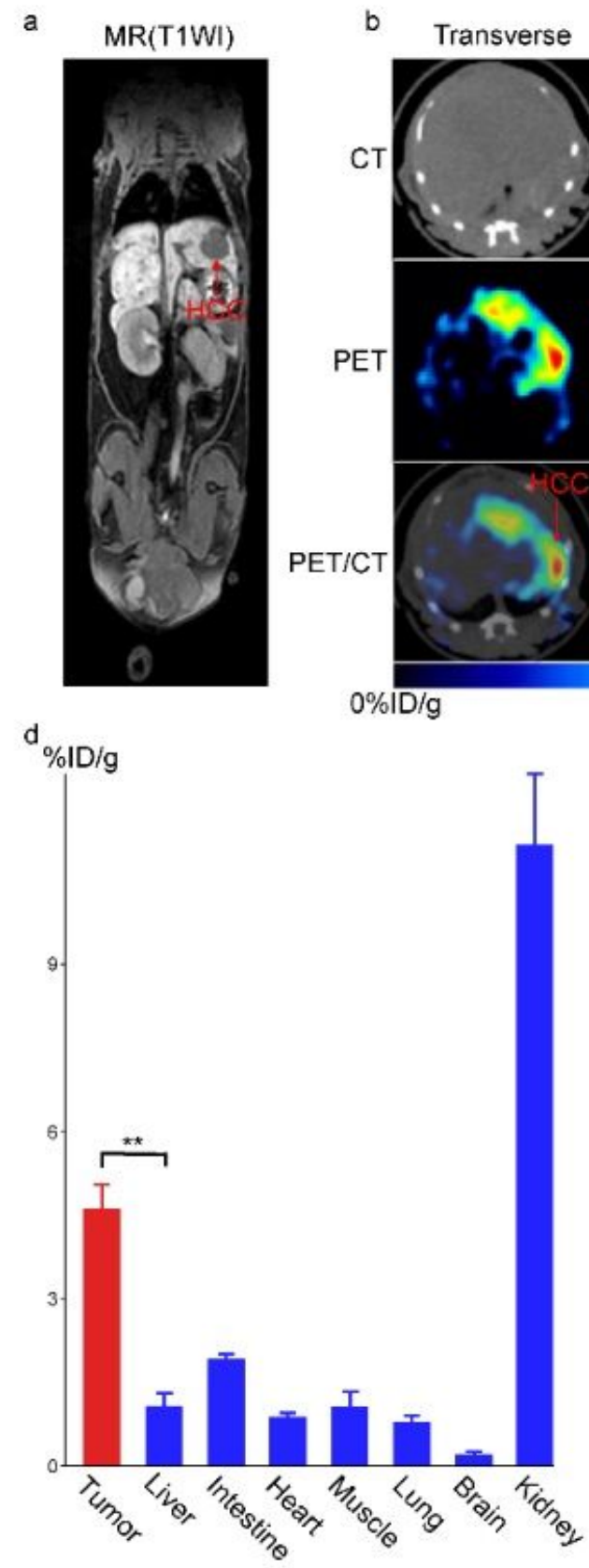
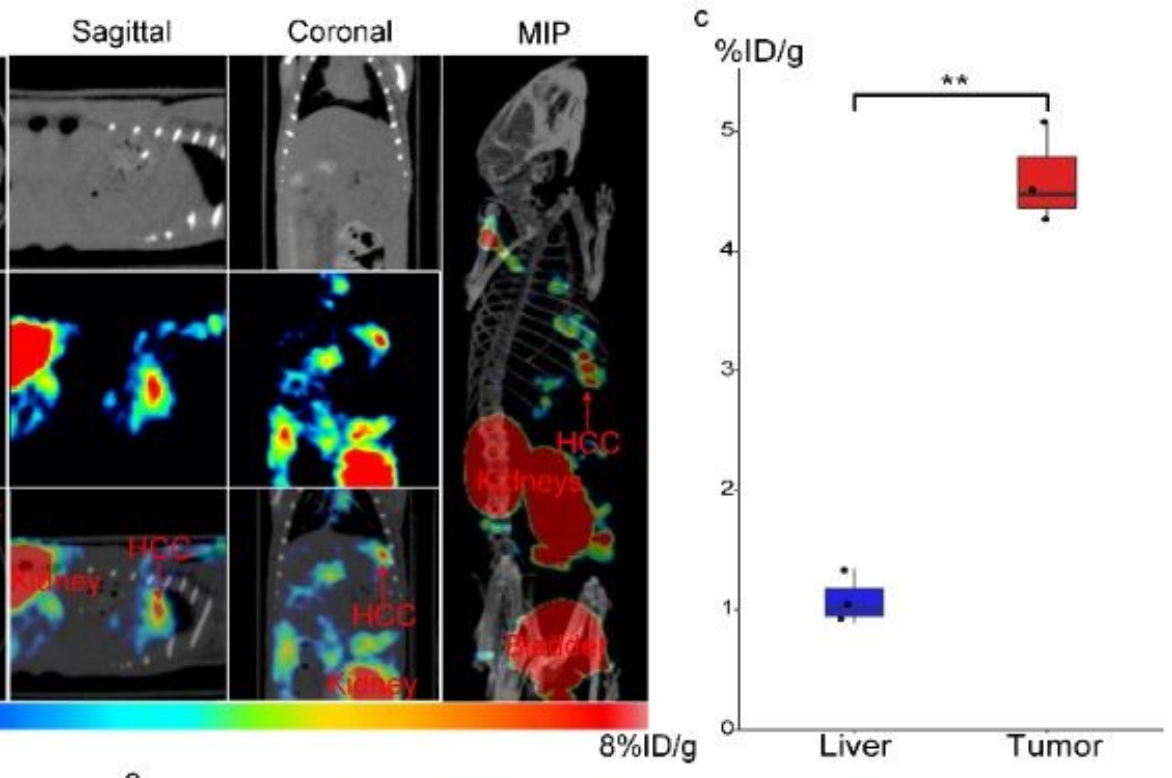

e



\section{Figure 4}

PET/CT imaging with 18F-S3 in DEN induced HCC mice. Representative MR(a) and PET/CT (b) imaging in the same mouse. (c) Quantification of tumor and liver uptakes of 18F-S3. Student's t-test, $\mathbb{\text { Q }} \mathrm{p}<.01, \mathrm{n}=$ 3 , mean \pm SD. (d) Uptake of 18F-S3 in tumor tissues and main organs at 90 min after injection $(n=3)$. Student's t-test, $\mathbb{X} \mathrm{p}<.01, \mathrm{n}=3$, mean $\pm \mathrm{SD}$. (e) NKAa1 expression was analyzed using immunohistochemistry (Scale bar, $100 \mu \mathrm{m}$ ) 

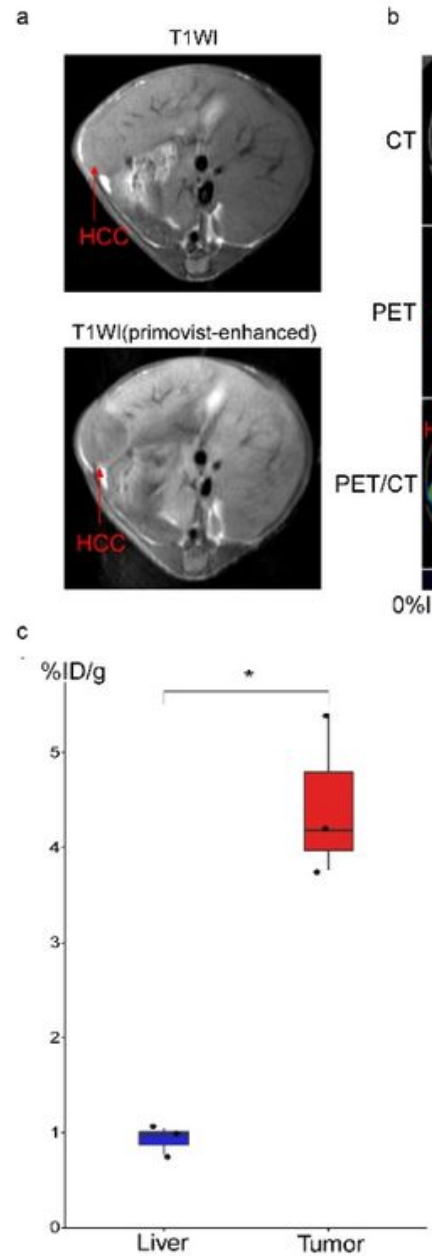
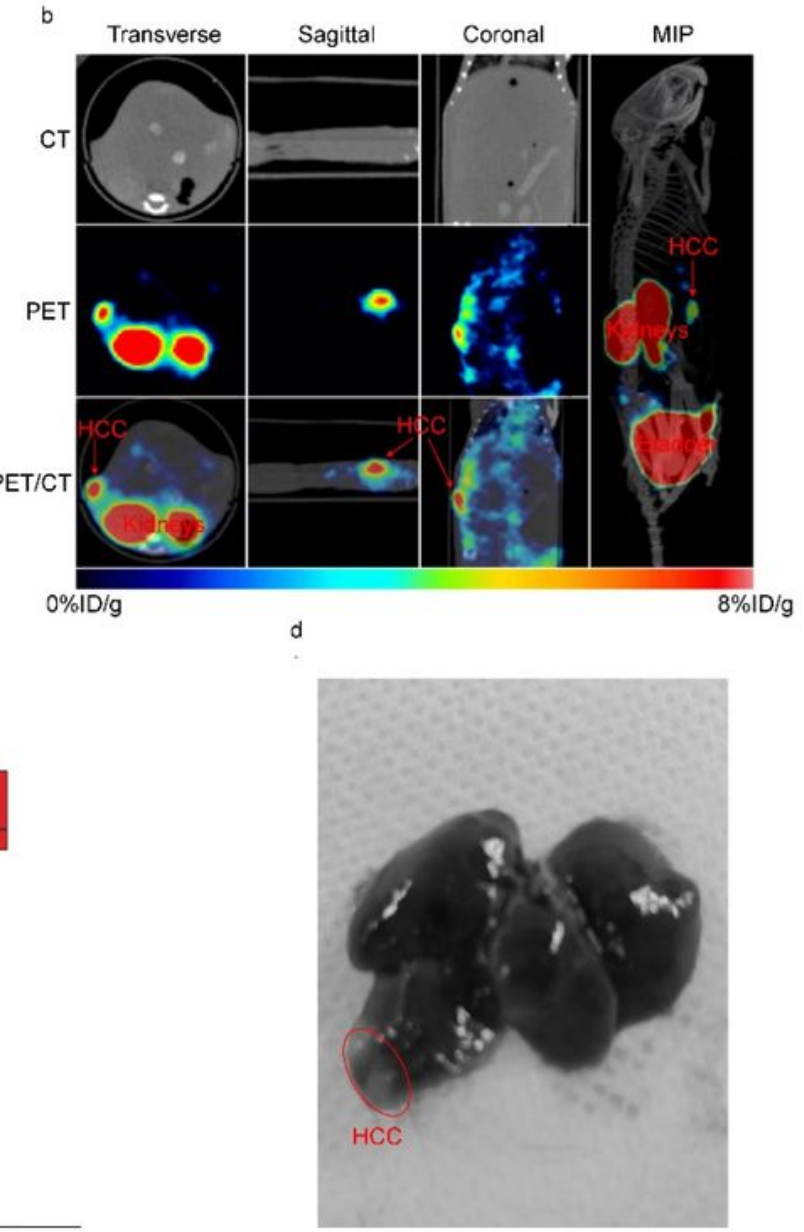

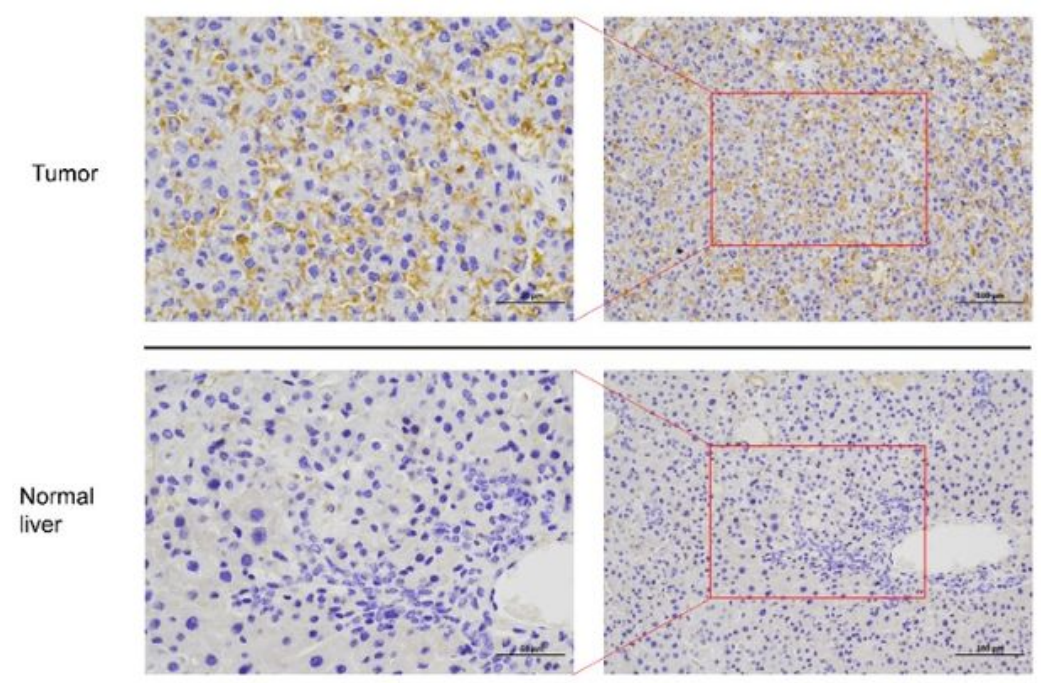

\section{Figure 5}

PET imaging with 18F-S3 in a genetically engineered HCC mouse model. MR(a) and PET/CT(b) imaging with 18F-S3 in the same mouse. (c) Quantification of tumor and liver uptakes of 18F-S3. Student's t-test, $\bigotimes p<.05, n=3$, mean \pm SD. (d) Photograph of the liver dissected. (e) NKAa1 expression was analyzed using immunohistochemistry (Scale bar, $100 \mu \mathrm{m}$ and $50 \mu \mathrm{m}$ ) 